\title{
PÁGINAS DE HISTÓRIA DA ENFERMAGEM: O J UBILEU DE OURO DE UMA OBRA (1951-2001)
}

History of Nursing Pages:

The Gold J ubilee of a Piece of Work (1951-2001)

Páginas de História de la Enfermería:

El J ubileo de Oro de una Obra (1951-2001)

AnaPaulacostaAlves

Osnir CaudianodaSilvajúrior

\section{Resumo}

Oobjeto desteestudo éa permanência do livo “Páginas deHstónia daEnfermagenXH Htónia da Enfermagem” em referêndias acadêmicas em2001, ano dbcinqüentenárioda primerira edição. Objetivo: Identificar a referênajazolivode

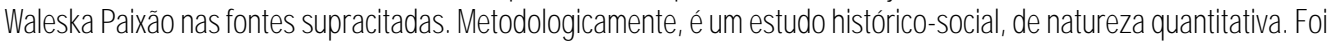
realizadb levantanerto bibliográico nes dissertaçães eteses detrês programas de pós-graduação, nos programas de discaplinadeH stóiiadaE Efermagememtrêsescolas públices degrad açãonomenicúpiodoRiodejaneiroeartigoscom

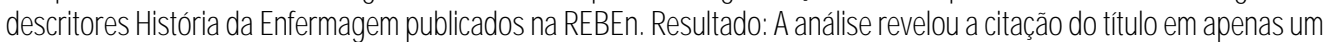
progranadedsciplinadegradlação. Condusão: Apesar caimportânajado referidblivo, devidbaoseupioneinistrmo Brasil (1951), as citações destadbraestãodesaparecendb nes referênias pesquisadas, oqueapontaparaosurginerto denovostítulos efortes paraoestudbdaH HóniadaEfermagem

Palauras-dhave HstóniadaEtermagem Ensino. PesquisaemEremagem

Abstract

The object of this study is the permanence of the book "Págines deHstóriadaE EremagenxH HstóriadaE ferma gem"', in 2001 academic references, celebratingfiftyyears of thefirst edition Theobjectiveis to identifya reference madeto WłleskaPaxãobookin thesources mentioned. It's asocia historicstudyas a thodblogy, fromquartitative neture Abibliographical suney wes nadeintexs and thesis fromtlree pos graduation programs, in the disaipline of Hstory of Nursing in the publicgraduationschools, inRio dejaneiroandartides with the keywordhistory of nursing publishedatRPEN Results: Theandysis shoustheditation of the book in only one graduation disaplines program Condusion: Despitetheimportance of relatedbook, pioner in Brazi (1951) theattions aredsappearinginthesearches references, what it poirt tothesprouting of newbooks and sourcesfor thestudy of thenursinghistory.

\section{Keymords \\ Nursing Hstory. Teading ResearchinNursing}

\section{Resumen}

Edjjetodeesteestudoes lapermanenciadal libro"Págines deHistóriadelaEnfermería/HistóriadelaEfermería" en referenias acadéricas en 2001, añodel anauentenariodela primeraedicón. I propósito es identificar lareferendaal libro de Valeska Paixão en las fuentes antedichas. Metodologicamente es un estudio histónico-sodal, de neturdezaa antitativa Fuéredizadbleartanieto bibliográfico enlasdisertacionesytesis detres programas deposgrado, enlosprogrames deladisaplinadeH HtoriadelaEtermería entres esaulas públicas depregradben e meniápiodel Rio dejaneroyartía los condescritores HstariadelaEvermería pudicados en la R⿴囗十En Resultados: E análisis reveló la atacón del título sólo en un programa de disciplina de graduacón Condusión Apesardelaimportandadel libroya rencionadb, dedidbal supionerismoen e Brasil (1951), las ditacionesdeestadbraestándesaparejendbenlasreferenias in vestigades, loqueapurtapara es surgimietodenuevostúlos yiuentes prael estudodelaH HstoriadelaErermería

Palabras dave HstoriadelaEfermería. Enseñanza InvestigacónenEfermería 
INTRODUÇÃO

O presente estudo inserese na linha de pesquisa "O Desenvolvimento da Enfermagemno Brasil" do Laboratónio de Pesquisa de Hstónia da Enfermagem (LAPHE) da EEAP/UNRO e emperha-se emverificar a importância do livro "História da Enfernagem", de WAleska Paixão e sua permanêndia como referência acadêmica para o estudo de Hstónia da Enfermagem decomidos os primeiros anqüenta anos de seu lançamento. Aautora foi professora de Éica e Hstónia da Enfermagemna Escola de ErfermagemAnna Nery da Universidade do Brasil, atual Universidade Federal do Rio de Janeiro, por onde publicau seu livro, lançado emprimera edição como títu “Páginas de Histónia da Evfernagem", em1951, e o fez porque sabia das dificuldades de professores e alunas, uma vez que a maior parte da literatura da época encontrava-se redigida eminglês - língua por eles desconhecida. Alémdisso, havia a questão da escassez e dispersão do material emnossa línguat.

No livro, a autora contempla a necessidade do conhecimento da história da profissão para maior compreensão da histónia da civilização e dos deveres que cabemà enfermeira e destaca três elementos que considera importantes e básicos no trabalho de uma profissional da Enfermagem a saber: espínito de senviço, habilidade e iêndia, considerando o primeiro o mais importante?

A história da Enfermagemfoi dividida emseis unidades, iniciando pelo Período antes de Cristo, Período da Unidade Gristã, passando pelo Período Gítico da Enfermageme primeiros movimentos de reforma da Enfermagem e, finalmente, Sistema Nightingale e Enfermagemno Brasil2.

Este estudo teve por motivação o contato coma obra "Histónia da Enfermagem" no primeiro período da graduação, quando esta fai apresentada cono una das referêndias bibliográficas para estudo, face à sua relevância na pesquisa emHstónia da Enfermagem e tambémpor ter sidb a autora una das pioneiras na pesquisa e difusão da Hstónia da Erfermagemno Brasil.

Sabese que durante mitos anos, o livro "Histónia da Enfermagem" foi o único utilizado para o ensino da Hstónia da Enfermagem Tomourse um best seller e, depois de 53 anos e inco edições, continua sendo apontadb informalmente cono referênia para o estudo e a pesquisa emHistória da Enfermagem Assim o objeto deste estudo é a permanência do livro "Páginas de Histónia da Enfermagem|Histónia da Enfermagem" em referências acadêmicas em 2001, ano do cinqüentenário da primeira edição. O objetivo é identificar a indicação do livro "História da Enfermagem", de Waleska Paixão, em referências acadênicas no ano de 2001, emarsos de graduação e pós-graduação na didade do Rio de Janeiro e publicações científicas na Revista Brasileira de Enfermagem(REBEn).

METODOLOGIA

Trata-se de uma pesquisa histórico-sodial, de natureza quantitativa do tipo análise teónico-empíica, uma análise preliminar da literatura sobre o tema e una busca limitada de informaçães doticas diretanerte no campo ou origem dos eventos pesquisados ${ }^{3}$. O estudo foi realizado por meio de levantamento bibliográfico nas dissertaçães e teses acadênicas em três programas de pós-graduação, nos programas de disciplina de Hstónia da Enfermagem nas três escolas públicas de graduação no muniápio do Rio de Janeiro e emartigos que apresentavamcomo palava-chave

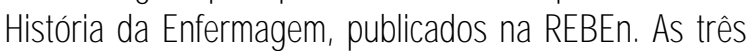
escolas pesquisadas foramnomeadas de $A$ B e C Os dados foramcoletadbs diretamente nos programas da disciplina Hstónia da Enfermagem da graduação, no aceno de dissertaçães e teses dos programas de pósgradação e no volume 54 (2001) da Revista Brasileira de Enfermagem no aceno da biblioteca setorial da Enfermagen/Nitrição do Centro de Giências Biológicas e da Saúde da Universidade Federal do Estado do Rio de Janeiro (UNRO) no rês de fevereiro do ano de 2004. As edições de "Páginas deH stónia da E fremagenx Históia da Enfermagem" pertencemao aceno das biblictecas da Escola de ErfermagemArna Nery/UFy e da Escola de ErfermagemAfredo Pinto/UNRO.

Os dados foram computados em instrumentos próprios, construídos pelos pesquisadores: una ficha comcélulas para o registro das ocorrências do título nos progranas das disciplinas de graduação e nos trabalhos de pós-graduação e outra para coleta de dados na Revista Brasileira de Enfermagem com células para registro do total de artigos, dos artigos com descritores História da Enfermageme para as citações do título buscado (anexo I Ae B).

A pesquisadora teve acesso aos programas de disciplina mediante carta de apresentação pelo orientador e solicitação de acesso aos programas das disciplinas aos professores responsáveis, indicados pelas Escolas que cederam cópias para o estudo (anevos II ellI). A pesquisa não foi subnetida ao Comitê de Ética emPesquisa por não se tratar de pesquisa com seres humanos. Os dados provêm de doamentação cedida pelos professores responsáveis 
pelas disciplinas nas escolas e faculdades, mediante carta de apresentação com os fins da pesquisa, mantidas sem identificação, e de documentos de acenos públicos, semindicação de sigilo ou restrição ao uso e edições do livro da autora emtela que igualmente fazem parte de acenvos de bibliotecas públicas. A fortion, cabe destacar que o artigo em análise em nenhummomento constrange, ofende, injuria ou causa maleficênda a qualquer individuo ou instituição direta ou indiretamente envolvidos.

Os dadbs biográficos da professora Włleska Paixão foramobtidos emdoa mentos encontrados na caixa

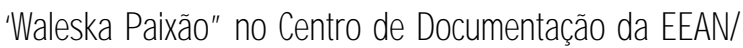
UTI, por meio de consulta direta emvisita agendada

A análise dos dados utilizourse da freqüência quantitativa das ditações nos doa mentos consultados e da interpretação do valor e da trajetória da obra para a enfermagembrasileira, particularmente para histónia da Enfermagem

DA AUTORA ${ }^{a}$

Utra pioneira nos estudos de História da Enfermagem ${ }^{4}$, VAleska Paixão nesceu em3 de noventbro de 1903, emPetrópdis, estadb do Rio de Janeiro. $\mathrm{Nb}$ ano de 1939, conduiu sua graduação na Escola de Enfermagem Carlos Chagas, emBelo Horizonte, Mnas Cerais. Alémdisso, arsou simittaneamente Sociologia, Filosofia e Mord, no Instituto Superior de Cutura Católica, emBelo Horizonte 5 .

Estudou na Universidade de Comell, emnbva lorque (Estadbs Unidos), no período de 1943-1944 onde arsou Administração e Ensino.

Lecionou emvárias instituições de ensino, entre elas: Colégio Sion, Colégio Santa Maria, Escola de Comércio de Belo Horizonte, Extemato Paixão, Escola de Enfermagem Carlos Chagas e Escola de EnfermagemAnna Nery. Nas três últimas, ocupou tambémo cargo de diretora.

Drigiu a Escola Carlos Chagas durante nove anos (1939-1948), o que aconteceu logo após a sua graduação, e a Escola Arna Nery durante dezesseis anos (1950-1966), emanbas substituindo Lás Netto dos Reys. Dentre as inúmeras funções exercidas por Włleska Paixão, destacase a presidênda da Associação Brasileira de Enfermagem(ABEn Nadiona) de 1950 a 1952, e das seçães do Rio de Janeiro, Sergipe e Mnes Cerais emperíodos distintos. Cupau, ainda, o cargo de vice-presidente internadional do OQAMG. (Comitê Internacional Católico de Enfermageme AssistentesMédico-Sociais), para a Anérica Latina de 1958 a 19626.

Após o seu pedido de aposentadoria, ocomido ao término do período de direção da Escola Arna Nery, a professora WAleska Paixão foi para o interior de Sergipe nummunípio a 49 quilômes de Aracaju comalta taxa de mortalidade infantil, Santa Rosa de Lima, um dos menores do Estado, comapenas 82 knª de área7, onde ficou por aproximadamente vinte anos. Lá estandb, a professora VAleska Paixão não só se preoaupou em dar assistência de enfermagem mas também se envolveu comoutras necessidades locais. Comisso, juntamente commis dies amiges, fundou, entre outras coisas, creches, jardimde infândia, escola primária e, ainda, organizou umsenviço médico-pediátricơ?.

Em1983, por meio da Escola de EnfermagemAma Nery, recebeu o título de 'Doctor Hbnoris Causa” pela Universidade Federal do Rio de Janeiro.

Interessante ressaltar a religiosicade na vica de Paixão. Catequista desde os 14 anos de idade?, sempre trabalhou embenefíio dos necessitados. Escreveu vérios artigos sobre esta tenática, como "Trabalho sobre Catequese", publicado na Revista dos Professores Católicos (1935), "Métodos e Programas" e "Formação da Consciênda", antos publicados nos Cademos Catequéticos (1936, 1940 e 1941, respectiventerte). Alémdisso, foi colaboradora do BoletimCatequético mo períodb de 1936 a 1950.

Eviqueceu a literatura infantil comalgumss naratives, conø "Contos da nimha Irmä" (1933) e "Contos para Mas Solbrimos" (1935), a lémde colaborar na revista infantil "OBeja For" (1920-1925) - contos

WAleska Paixão faleceu em 25 de noverbro de 19936, aos 90 anos.

\section{DO LIVRO}

A primeira edção do livro "Hstória da Enfermagem” foi publicada em1951 pela Universidade do Brasil. A segunda (1960), terceira (1963) e quarta (1968) edições forampublicadas pelo editor Bruno Bucaini. A quinta e útima edição fai publicada em1979, pela Júlio C Reis Livaria Cabe ressaltar que o título "Hstória da Enfermagem" somente foi utilizado a partir da quarta edição, sendo as três primeiras normadas de "Páginas de Histónia da Enfermagem".

Tratase do primero livro, escrito no Brasil, sobre a histónia da Enfermageme foi, durante mitos anos, o único a ser uilizado mo ensino e apoio à pesquisa em Hstónia da Enfermagem

Paixão, ao descrever a história da Enfermagem preoaupourse emregistrála desde os tempos antigos por acreditar que a histónia da profissão está intimamente ligada à história das civilizações.

O livro é estruturado emuma visão tradicional da história; ou seja, os fatos são narrados em ordem 
crondlógica dos aconteainertos, de onde são destacados personagens de notória importânia histórica emcada períodb descrito. Aquinta e mais recente edição (1979) temo formo de 13,5 × 20 ancom141 págines.

Whleska Paixão relata que no período antes de Gisto, os conheaimetos dentíficos estavam, na maioria das vezes, associados às práticas religiosas e ressalta a figura de Hpócrates, na Grédia que lançou as bases da medicina dentúfica. Destaca umenome progresso na área da saúde coma construção de hospitais e abrigos no Período da Unidade Cristã, nos quais a assistênia era concedida por diáconos e diaconisas, assimcono por viúves, virgens e damas da sociedade E ainda, o valioso papel de São Frandisco de Assis, séalo XII, na fundação das ordens frandiscanas.

No Período Grítico da Enfermagem determinado pela reforma religiosa do século XM, na Europa, ocorreu o fechamento de inúmeros hospitais, devidb à expulsão das religiosas dessas instituições e sua conseqüente substituçãa por pessoas, segundo Waleska, de duvidosa moralidade, enquanto, nos primeiros movimentos de reforma da Enfermagem distinguiurse a realização de obras de caridade por S. Vicente de Paulo, criando a Confraria da Caridade e, posteriomente, o Instituto das Filhas da Caridade (Imãs de Caridade), juntamente comluiza de Marillac

Paixão põe em destaque, na unidade Sistema Nightingale, a grande figura de Forence Nightingale, sua trajetória e suas conquistas. Salienta, indusive, a criação de algumas associações de dasse e a participação ativa da Curz Vermelha em vários momentos da reforma da Enfermagem

Na pate referente à Evfermagemno Brasil, aborda a fundação dos primeros hospitais e escolas de enfermagemno pás, e, por fim a autora destaca duas notáveis personalidades da nedína sanitarista: Osualdb Cuzz, responsável, entre outras coisas, pela camparha de combate à Fedre Anrrela e a vedinação contra a Varída no Rio de Janeiro, e Carlos Chagas, diretor do Departamento Nacional de Saúde Púdlica e fundador da Escola de ErfermagemArna Nery.

A perspectiva do texto é a apresentação tradicional da "grande histónia" que remonta às fronteiras da pré história, onde a Enfermagem é associada ao sertinerto de presenação da espédie ou aos instintos maternos para coma cria. Dáí, emuma seqüência temporal que se inicia na antiguidade oriental, avença temporal e geograficamente e mostra os avanços e retrocessos, lidos comtintas de selvageria-barbárie duilizacãa ${ }^{10}$. Emtodb o texto, sobressai a importânaia atribuída ao sentimento religioso cristão como instrumento de evolução positiva da Enfermagem
Tantbémestão res vérizs edç̃és do lino os aspectos de datrinação nord emeremplos de dedcação heráica àcausa da Evfermagemde seus líderes e expoentes, não só palo corhedinerto dertífico, nas tambémpela alonegação, por vezes missionária Nâo há contradições internas aos personagens nemra forma conro se deserrola a história

RESULTADOS E DISCUSSÃO

O exame dos programas de pós-graduação revelou que no programa $A$ das 21 dissertacões apresentadas no ano de 2001, o assunto Hstónia da Effermagemfoi contemplado emapenes una delas. Porém, não houve citação do livro emestudo. No programa B, das 40 dissertações e teses defendidas no referidb ano, foram localizadas 5 de Histónia da Enfermagem entretanto, não houve alusões à obra. No prograna $C$, não foi identificada nenhuma dissertação de História da Enfermagemdentre as 2 condú́das do resmo ano. Assim dos 63 estudbs de Pós-graduação strico sensu condúdos em2001, nerhumaitou o livro "História da Enfermagem" como referêndia.

Quarto aos programas de disaiplina de Histónia da Enfermagem (graduação), emapenas una das escolas (escola A) foi encontrada menção ao livro. Nas demais escolas (B e O, não foramidentificadas ditações do livro de Włleska Paixão. Cabe destacar que, na escola $B$, a disciplina Histónia da Enfermagemé ministrada emvários momentos. A pesquisadora teve acesso a apenas umdestes programas de disciplina, no qual não houve referênia ao livro "Hstónia da Erfermagem”.

A análise dos 65 artigos da Revista Brasileira de Enfermagempublicados em2001 revelou que rẽo houve referênia ao livro "Histónia da Enfermagem" nos 17 trabalhos comdescritores Hstónia da Enfermagem

Cabe chamar a atenção para o fato de que o $n ? 2$, abr.jun 2001, fai o número comermorativo dos 75 anos da ABEn (1926-2001), entidade onde a autora emtela manteve longa e destacada atuação.

A análise do livro, como novo dgjeto da histónia, na leitura deChatier eRoche, destocarseda "histónia literária ligada às grandes doras e conduridas por isso a considerar o livro enquanto portador da novidade estética ou intelectual" para uma análise quantitativa, para "o prodlenta des influências, quer fossemda sua época sobre o escritor au aquela da dora sobre seu púdico". Emnosso neio acadêmico atul, adbtanos a idéa de que a métipla ocorênaia de umautor au dora representa o impacto ea importânaia destes para o campo dentífico"1.

No processo de consolidação da Enfermagemcono campo de saber universitário, a importânda da produção de textos publicados emlivros representou umdesafio para as pioneiras, dentre as quais se insere 
a professora Włleska Paixão, momente tratandb-se da história da Enfermagem que se incumbia de escrever a vercade dos fatos, dar continuidade a una tradição e mesmo de ciála onde não existia. Para citar apenas dois elementos: abrir um mercado editorial e distribuir livros de enfermagemnacionais pelo Brasil. Estas dfiauldades, juntamente coma idáa reinante no senso commde que a histónia, una vez contada e escrita, rẽo precisava de noves interpretações, certamente contribuíram para a longa permanênda solitária de "Páginas de História da Enfermagem" no setor de Hstónia da Enfermagemnes estantes.

Dada a natureza quantitativa deste estudo, pudemos identificar a baixa ocorrênia do título em estudo nos progranas de graduação em Enfermagem no muiápio do Rio de Janeiro e a parca utilização na produção científica de História da Enfermagemnos artigos e produtos de estudos de pós-graduação strictosensu pesquisados.

CONCLUSÃO

Emque pese a indisatível importância do livro “Hstónia da Enfermagem”, de Vłleska Paixão, devido ao seu pioneinistro (1951) e pelo fato de este ter sido durante mitos anos o único livro de Hstónia da Enfermagemeditado no Brasil, as citações desta dora foramraras nes referêndas pesquisadas, o que aponta

\section{Referências}

1. PaixãoWPágines dehistóniadaenfemagem Riodejaneiro(Pl): UniversidadedoBrasil; 1951.

$2 . \quad H i t o ́ n i a d a$ enfermagem 5 a ed Riodejaneiro(R)): JúlioC Rés; 1979.

3. TadizamaT, Mendes G Comofazer monografianaprática $\wp^{a} e d$. RiodeJanero(BI): EdFG/, 2001

4. BarreiralA BaptistaSS Nexos entreapesquisaemhistóniada effermagemeo processo decientifização da profissão. Anaisdb $51^{\circ}$ Congresso Brasileiro de Enfermagem $10^{\circ}$ Congresso de Erfermería; 1999 aut2-7; Florianópolis (SQ, Brasil, Florianópolis (SO: ABEr, 2000. p.295-311.

5. Coelho P. AEscola de EnfermagemArna Nery sua histónia, nossas menớrias. Riodejaneiro(B)): Cutura Médica; 1997.

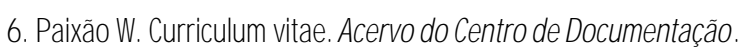
RiodeJaneiro(B):: UPJ/ EAN 1978.

7. Cóes CSRL SantaRosadelina. [Online] [Ctadb 19ador 2004] Disponivel em http://wwwinfonet.combr/anformmuicipios/ meniapio_linahtm para o surgimento de novos títulos e fortes para o estudo da Histónia da Enfermagem possivelmente prodito de novos estudos empreendidos nos grupos de pesquisa de Hstónia da Enfermagem como o Núdeo de Pesquisa de História da Enfermagem Brasileira (1993) $)^{12}$ e o Laboratónio de Pesquisa de Hstónia da Enfermagem $(2000)^{13}$, que sustentam linhas de pesquisa na área de Hstónia da Enfermagem, comprodução de livros e artigos emperiódicos. Estes estudos caraderizamse, de modb geral, pelo recorte de djjetos na histónia da Enfermagem emvez da construção da “Grande Histónia da Effermagem”, emuma perspectiva totalizante emgrandes espaços temporais e geográficos comens às abordagens tradicionais.

Cabe destacar que este estudb, ao terpo emque valoriza a personageme a obra, indiscutivelmente relevantes para o ensino e pesquisa emHistónia da Enfermagem destinase tambémà divigação do tera às novas geraçães e à iniciação científica emHstónia da Enfermagemno âmbito da graduação ${ }^{14}$.

Por último e não menos importante, a Hstória da Enfermagemé umterreno altamente feando para a ampliação do capital altural de estudantes de gradtr ação e una forma de inicálos na pesquisa e no com promisso comos valores da profissão, desde os primeiros períodos. A "pequena contribuição" de que fa lava a autora, como una semente, frutificou.

8. PaixãoWEtrevista AcenodoCentrodeDoamentação. Rio de Janeiro(P):: UFI/ $\mp A N$ [ 198-?].

9. . Hbje mais do que ontem Rio de Janero (R)): PAT/ Senviços eProduções Gráficos; [1987].

10. Cardoso CF. Hstóniaeparadignæsivivis In Cardbso CF, VairfesR orgarizadbres. Donínios dahistónia ensaios deteoriaen todbloga RiodeJaneiro(R)): Campus; 1997. p1-23.

11. Chartier R Roche D Olivo: unamudançadeperspectiva. In Le Coff], NoraP, orgarizadores. Hstória novos djjetos. Riodejaneiro (B)): FranciscoAlves; 1995. p. 99-115.

12. La rençoLHBC, QiveralCS, Sattos TCF. NúdeodePesquisade HstóriadaEnermagemBrasileira. EscAmaNeryRE Rerm1997ju; 1(resp): 99-102

13. Silva Júnior $\propto$, Mreira A Porto $F R$ AnrorimWM Brito I. Labordtóniodepesquisadehistóniacaerfermagem histónia, menớria erelíquizs-umparocalahistóniadaerfermagemo Brasil. Revistade Pesquisa aidadbéfundanenta 2001; 5(2):78-85.

14. Barreira IA Contribuições da históriada enfermagemparao desenvolvimento da profissão. Esc Anna Nery R Enferm1999 abr, 3(1): 125-41. 
Notas

a AgradecenrosàProfẹ. D. - VuinaLanzarinideCandho, professora aposertada da Escola de EvfermagemAnna Nery/UFI, pelas informações gentilmenteformecidas embrevecontato, naEscolade ErfermagemAlfredo Pinto/UNRIOem2003.

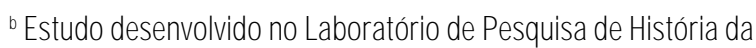
EvfermagemdaEscoladeEfermagemAfredbPinto/UNROTrabalho premiadb comoterceirolugar na 7-jomada Nacional deH stóniada Enfermagenx11으 Pesquisando emEnfermagem da Escola de EfermagemAmaNky/LPI. 2004.

\section{Sobre os Autores}

Arapalacostanives

Acadênicadb 70̣períodbdegraduaçãodaEAP/UNRIO

MentbrodoLAPIE

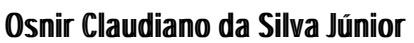

Professor Adiunto db DepartanertodeE EfermagemFundanental daEAP/UNRIO. Dator emE Efermagem Pesquisadordb LAPIE Oientador. 